\title{
Dermoscopy of Dermatofibrosarcoma Protuberans: What Do We Know?
}

\author{
Gabriela F. Escobar ${ }^{1}$, Caroline K. Ribeiro ${ }^{2}$, Leandro L. Leite ${ }^{1}$, \\ Carolina R. Barone ${ }^{1}$, André Cartell ${ }^{2}$
}

1 Department of Dermatology, Hospital de Clínicas de Porto Alegre (HCPA), Brazil
2 Medical School, University of Rio Grande do Sul (UFRGS), Brazil
3 Department of Pathology, Hospital de Clínicas de Porto Alegre (HCPA), Brazil

Key words: dermatofibrosarcoma protuberans, dermoscopy, nonmelanocytic skin cancer, malignant cutaneous tumor, soft tissue tumor

Citation: Escobar GF, Ribeiro CK, Leite LL, Barone CR, Cartell A. Dermoscopy of dermatofibrosarcoma protuberans: what do we know? Dermatol Pract Concept. 2019;9(2):139-145. DOI: https://doi.org/10.5826/dpc.0902a10

Accepted: February 21, 2019; Published: April 30, 2019

Copyright: (02019 Fortes Escobar et al. This is an open-access article distributed under the terms of the Creative Commons Attribution License, which permits unrestricted use, distribution, and reproduction in any medium, provided the original author and source are credited.

Funding: None.

Competing interests: The authors have no conflicts of interest to disclose.

Authorship: All authors have contributed significantly to this publication.

Corresponding author: Gabriela Fortes Escobar, MD, MSc, Rua Ramiro Barcelos, 2350, 90035-903 Porto Alegre, RS, Brazil. Email: gescobar@hcpa.edu.br

ABSTRACT Background: Dermatofibrosarcoma protuberans (DFSP) is an uncommon mesenchymal tumor of intermediate malignancy. Its rarity and slow progression often imply a delayed diagnosis. There are few previous reports of dermoscopic features of DFSP and most are single case descriptions.

Objectives: To report 2 cases of DFSP and their dermoscopic features, as well as conduct a review of all previous cases published addressing the use of dermoscopy in this tumor.

Methods: We conducted a literature search for all dermoscopic cases of DFSP. In addition, we presented 2 additional cases and compared them to the earlier findings.

Results: We summarized the main dermoscopic findings of DFSP based on analysis from 32 patients. The most common features of this tumor are the presence of vessels $(81 \%)$, followed by a pigmented network $(78 \%)$ and a pinkish background $(66 \%)$.

Conclusions: DFSP can mimic benign lesions and the diagnosis may be challenging. Dermoscopy is an important tool that may enhance clinical suspicion toward the diagnosis of DFSP.

\section{Introduction}

Dermatofibrosarcoma protuberans (DFSP) is a fibrohistiocytic tumor that arises from the dermis and invades deeper tissue [1,2]. Classic DFSP clinically appears as an indurated plaque or nodule, exhibiting flesh to reddish brown color [1].
The tumor is locally aggressive and has a high rate of recurrence; however, it rarely metastasizes [1,2]. The diagnosis of DFSP is often delayed because of its rarity, slow progression, and lack of early clinical clues. Therefore, dermoscopy may be a valuable tool in the recognition and prompt diagnosis of this tumor. Few previous reports have addressed the dermo- 


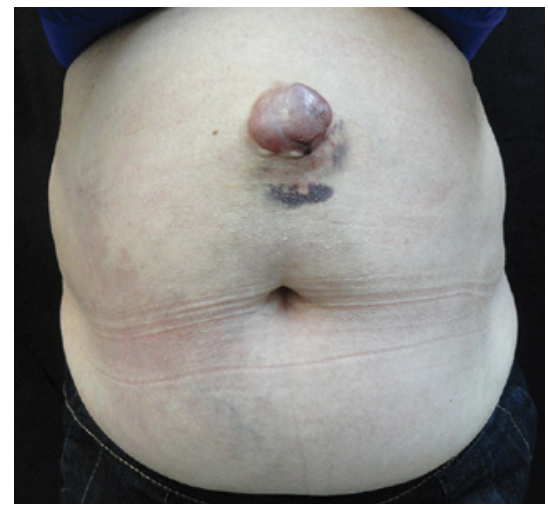

Figure 1. Erythematous tumor located on the abdomen. An ecchymotic hue post-biopsy could be visualized. [Copyright: (O2019 Escobar et al.]

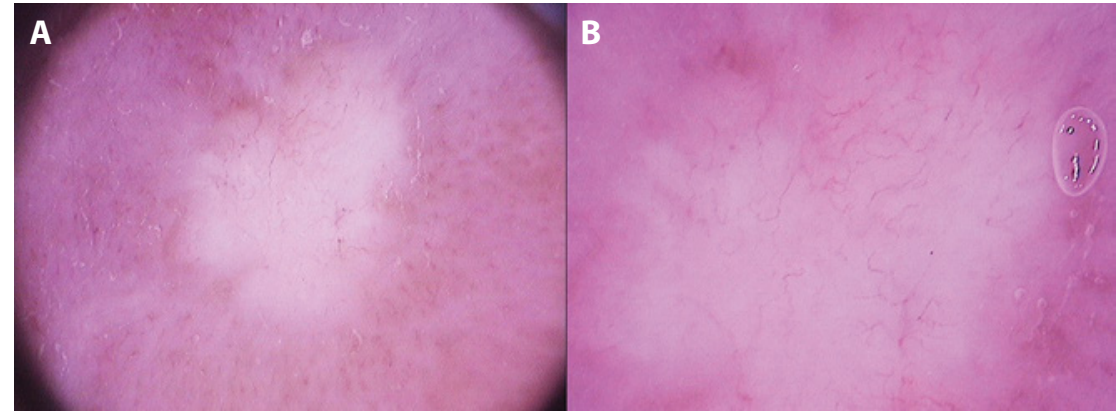

Figure 2. (A) Fine arborizing telangiectasias and linear vessels over structureless depigmented areas, surrounded by a pink background with unfocused vessels $(\times 20)$. (B) Close-up to visualize the central vascular structures $(\times 50)$. [Copyright: (2019 Escobar et al.]

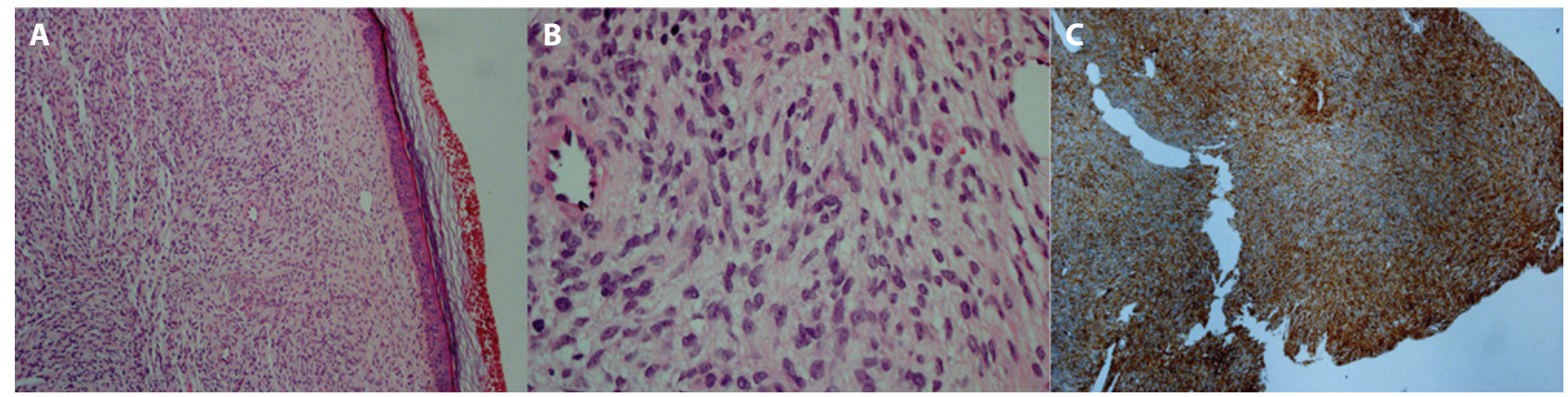

Figure 3. (A) Spindle-shaped cells arranged in a storiform pattern $(H \& E, \times 100)$. (B) $(H \& E, \times 400)$. (C) Immunohistochemistry using antiCD34 antibody revealed that spindle cells were positive. H\&E = hematoxylin and eosin. [Copyright: (2019 Escobar et al.]

scopic aspects of DFSP and its variants [3-11]. In this article, we present 2 cases of DFSP and their dermoscopic features, as well as a review of previously published dermoscopic descriptions of this rare neoplasm.

\section{Case Reports}

\section{Patient 1}

A 73-year-old woman, Fitzpatrick skin phototype III, presented with a 5 -year history of an asymptomatic, slowly enlarging, nodular lesion located on the abdomen. Physical examination showed a solitary mesogastric erythematous tumor measuring $80 \times 80 \mathrm{~mm}$ (Figure 1). On palpation, the lesion was firm and not tender. Dermoscopy revealed fine arborizing telangiectasias and linear vessels over structureless depigmented areas, surrounded by a pink background with unfocused vessels (Figure 2, A and B). A 5-mm punch biopsy was performed. Histopathological examination revealed spindle-shaped tumor cells in a storiform pattern (Figure 3, A and B), suggesting DFSP. The diagnosis was confirmed by immunohistochemistry, with positive staining using antiCD34 antibody (Figure 3C). Magnetic resonance imaging showed a homogeneous nodular lesion circumscribed to the subcutaneous tissue and a chest $\mathrm{x}$-ray ruled out lung metas- tasis. The lesion was totally excised with $3-\mathrm{cm}$ margins and there has been no recurrence in 2 years of follow-up.

\section{Patient 2}

A 50-year-old woman, Fitzpatrick skin phototype IV, was referred to the dermatology department to evaluate the possibility of Cowden syndrome, since she had been previously diagnosed with breast cancer and papillary thyroid carcinoma. After a thorough physical examination, the patient did not meet the clinical diagnostic criteria for the syndrome. However, an inframammary brownish plaque lesion was observed. There was no history of trauma or previous procedures to the area. The patient had noticed a gradual enlargement of the lesion in the preceding 2 years. Physical examination showed a brown atrophic plaque on the left inframammary region, measuring $25 \times 20 \mathrm{~mm}$ (Figure 4). On palpation, the lesion was indurated and tender and resembled a morphea plaque. Dermoscopy revealed a pigmented network, with areas with interruptions in the network, over a pinkish background. No vascular structures were visible (Figure 5, A and B). A 5-mm punch biopsy was performed. Histopathological examination revealed a storiform pattern of a dermal spindle cell proliferation infiltrating the subcutaneous fat tissue in a honeycomb pattern (Figure 6A). Immunohistochemistry using anti-CD34 


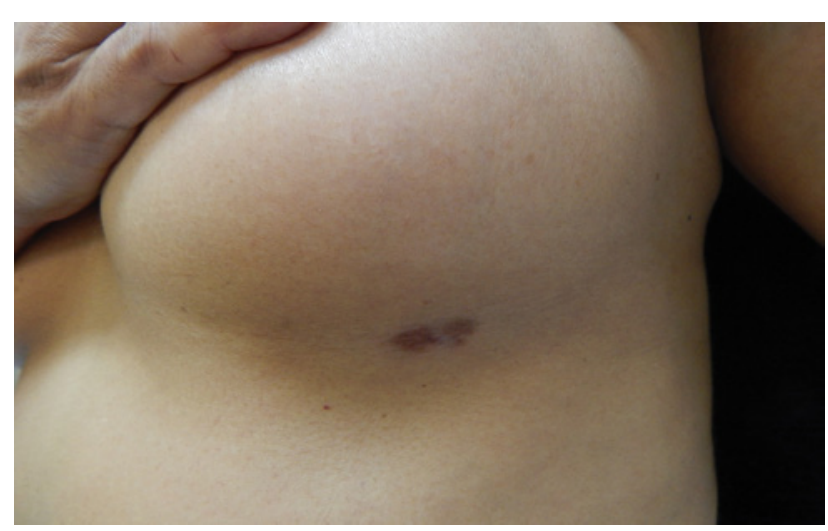

Figure 4. Brown atrophic plaque on the left inframammary region. [Copyright: (C2019 Escobar et al.]

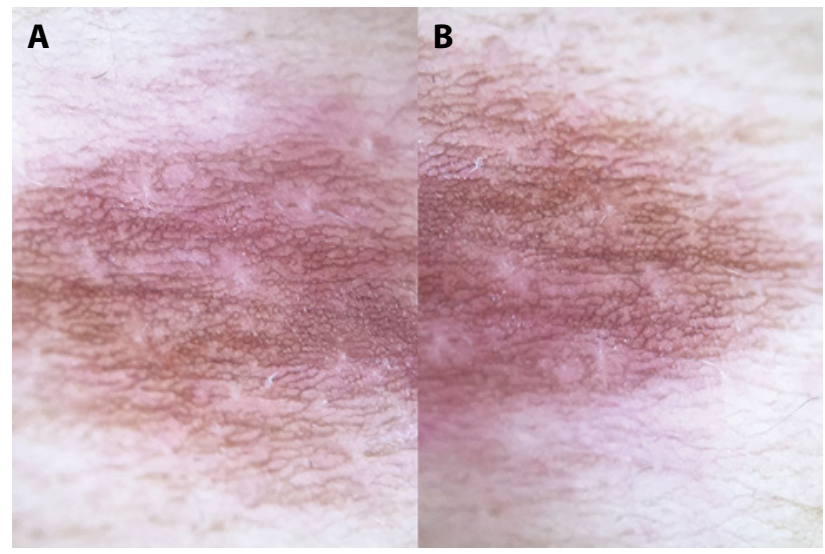

Figure 5. (A,B) Pigmented network, with areas with interruptions in the network, over a pinkish background. No vascular structures were visible $(\times 20)$. [Copyright: (2019 Escobar et al.]
Figure 6. (A) Spindle-shaped cells arranged in a storiform pattern (H\&E, $\times 100)$. (B) Immunohistochemistry using antiCD34 antibody revealed that spindle cells were positive. H\&E $=$ hematoxylin and eosin. [Copyright: @2019 Escobar et al.]

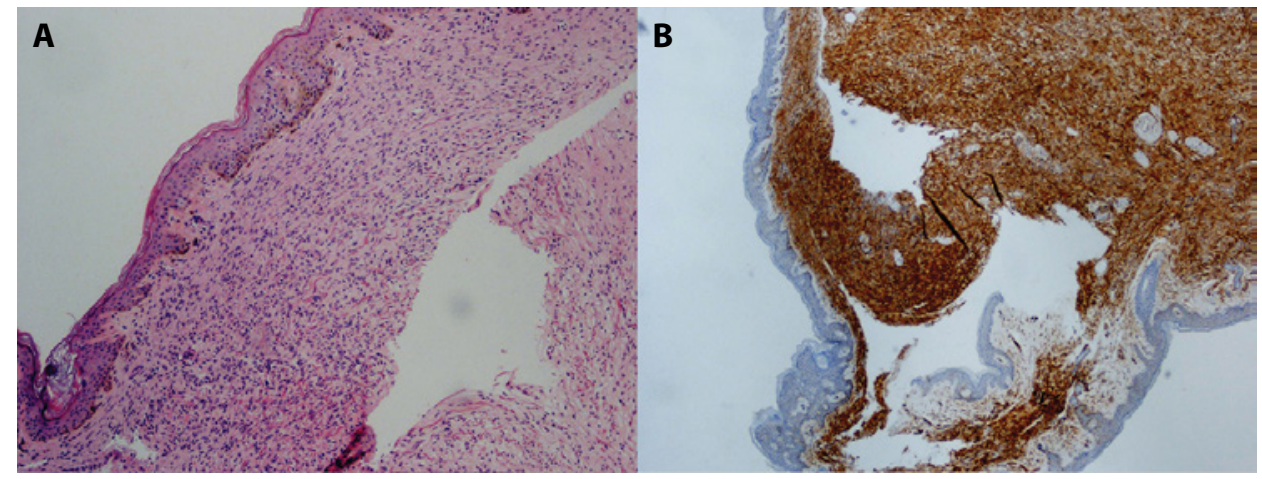

antibody revealed that spindle cells were positive, confirming the diagnosis of DFSP (Figure 6B). The lesion was totally excised with margins of $4 \mathrm{~cm}$ and there has been no recurrence in 2 years of follow-up.

\section{Discussion}

DFSP is a slowly growing mesenchymal neoplasm, characterized by high morbidity due to its local invasion and high recurrence rate after surgical excision $[12,13]$. The peak age at onset is between 20 and 50 years, and incidence among women is 1.14 times higher than in men [14]. The tumor usually presents as a slowly growing, painless, reddish brown nodule or plaque. The trunk is the most common anatomic site, followed by proximal extremities $[1,15]$. Furthermore, DFSP may present as a morphea-like, atrophic, sclerotic, violaceous plaque that may ulcerate as it gradually increases in size $[2,11]$. The main differential diagnoses include cutaneous melanoma and dermatological manifestations of metastatic carcinomas. Also, DFSP may mimic benign lesions such as dermatofibromas, hypertrophic scars, and keloids [2,9,11].

A chromosome translocation is found in more than $90 \%$ of the cases, involving chromosomes $17 \mathrm{q} 22$ and 22q13 [1,2]. The constitutively activated platelet-derived growth factor- $\beta$
(PDGFB) signaling pathway plays a central role in the proliferation of DFSP tumor cells [2]. The typical histopathological characteristics of DFSP are described as uniform spindle cells with elongated nuclei, showing minimal atypia or mitotic activity, resulting in a storiform pattern [2,12]. The tumor infiltrates into the subcutaneous tissue, creating the characteristic honeycomb pattern. Other spindle cell tumors such as dermatofibroma, malignant fibrous histiocytoma, atypical fibroxanthoma, desmoplastic melanoma, Kaposi sarcoma, and solitary fibrous tumor are the histological differential diagnoses [2,12]. The infiltrative pattern of DFSP helps to differentiate it from benign entities, even though it is usually not sufficient for a conclusive diagnosis. In these cases, immunohistochemistry becomes a useful resource, since DFSP reveals positivity for CD34 and negativity for S100 protein, factor XIIIA, and desmin [1,2]. Histological variants of DFSP have been described in the literature, such as the pigmented variant (Bednar tumor), myxoid DFSP, juvenile form (giant cell fibroblastoma), fibrosarcomatous variant, sclerosing DFSP, and atrophic subtype [16,17].

Dermoscopic findings of DFSP are limited in the literature [3-11]. We performed an extensive search in the PubMed database and found a total of 9 articles with 30 patients (Table 1). The first report, conducted by Bernard et al [3], 


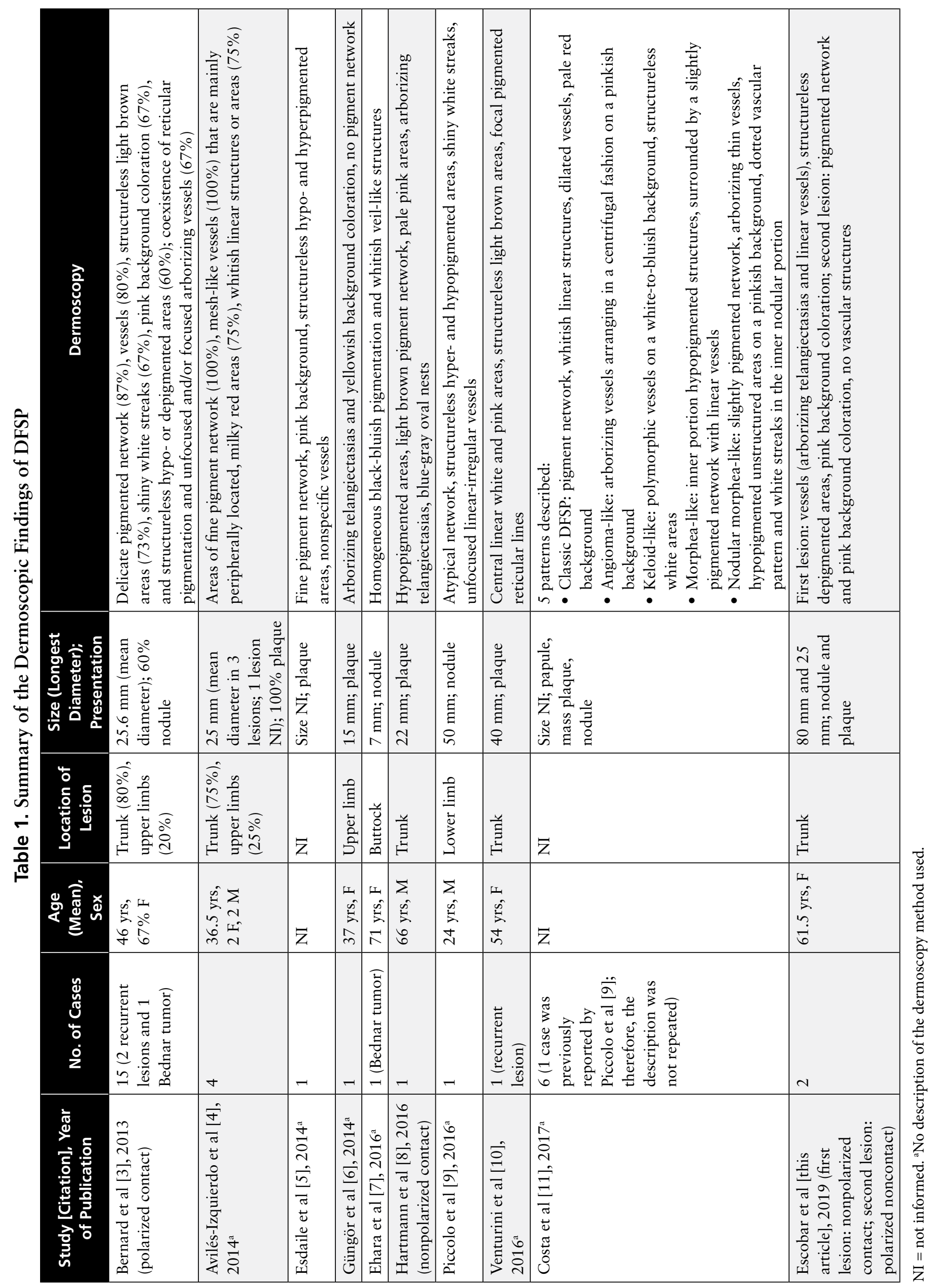


analyzed 15 cases of DFSP, including 1 case of Bednar tumor. In this study, $80 \%$ of the lesions were located on the trunk, while the remaining were on the upper limbs. In this article, 6 dermoscopic characteristics and their frequency were described: delicate pigmented network (87\%), vessels (80\%), structureless light brown areas $(73 \%)$, shiny white streaks (67\%), pink background coloration (67\%), and structureless hypo- or depigmented areas $(60 \%)$. Among the lesions with vessels, all had unfocused vessels and half had a mixture of unfocused and focused vessels. Arborizing vessels were the most prevalent vascular structure, since it was found in 11 of 12 cases. Therefore, the authors concluded that the most common dermoscopic pattern observed was the coexistence of reticular pigmentation and unfocused and/or focused arborizing vessels. Another interesting finding was the high number of dermoscopic features per lesion (a median of 4), also called a multicomponent pattern, which is suggestive of malignant tumors $[3,18,19]$.

Subsequently, Avilés-Izquierdo et al [4] described the dermoscopic features in 4 cases of DFSP. All lesions consisted of plaques, located mainly on the trunk (75\%). The most prominent dermoscopic features observed by the authors were mesh-like vessels, mostly located peripherally, which histologically corresponded to dilated vessels in the superficial dermis. In addition, all cases presented with a fine pigment network, histologically represented by hyperpigmentation in the basal cell layer. Milky red areas were observed in 3 cases, as well as whitish linear structures or areas, which histologically corresponded to intersecting bands of tumor cells in the dermis, forming a storiform pattern. Esdaile et al [5] also described a similar case with a fine pigment network over a pink background, with structureless hypo- and hyperpigmented areas and nonspecific vessels.

A further report by Güngör et al [6] described 1 case of the atrophic variant of DFSP. In this patient, dermoscopic examination revealed arborizing telangiectasias and a yellowish background. The authors suggested that this particular background coloration could be explained by dermal atrophy and the close approximation of the subcutis to the epidermis. In addition, Ehara et al [7] described 1 case of the pigmented variant of DFSP (Bednar tumor), which clinically and dermoscopically mimicked a blue nevus. In this case, a homogeneous black-bluish pigmentation with white-veil structures was observed, while no vessels could be visualized. While the diffuse and intense pigmentation was attributed to melanin-laden spindle cells in the dermis and subcutaneous tissue, the white veil structures corresponded to collagen in the upper dermis. Since the tumor had a deeper presentation, no vascular structures could be appreciated, which differed from the Bednar case reported by Bernard et al, which displayed vessels on dermoscopy [3].
Furthermore, Hartmann et al [8] described a case of DFSP with clinical and dermoscopic features that resembled a basal cell carcinoma. In this case, dermoscopy showed 2 criteria used to diagnose basal cell carcinoma: arborizing telangiectasias and structures similar to blue-gray ovoid nests. In addition, hypopigmented areas, light brown pigment network, and pale pink areas could also be visualized. DFSP may also resemble keloids, particularly in patients with darker skin. Piccolo et al [9] described the case of an African male patient with the following dermoscopic features: atypical network, structureless hyper- and hypopigmented areas, shiny white streaks, and unfocused linear-irregular vessels. This report also highlights the dermoscopic multicomponent pattern, which should always raise a red flag for malignancy.

Recurrent DFSP may be very challenging to diagnose because it can resemble scar tissue. Venturini et al [10] reported 1 case in which they observed linear white and pink areas surrounded by structureless light brown areas with focal pigmented reticular lines. These findings were similar to those described by Bernard et al [3]. This article included 2 recurrent lesions and the authors did not identify differences between the dermoscopic patterns observed in patients with recurrent DFSP and those with primary lesions.

Recently, Costa et al [11] analyzed dermoscopic findings in 5 clinical variants of DFSP (however, 1 was previously described [9]). The angioma-like DFSP displayed thick arborizing vessels arranged in a centrifugal fashion on a pinkish background. Also presenting with vascular structures, the keloid-like DFSP had polymorphic vessels (linear and arborizing) on a white-to-bluish background and structureless white areas. Moreover, a morphea-like plaque DFSP was reported, exhibiting a slightly pigmented network, structures, and linear vessels. However, nodule-plaque morphea-like DFSP presented with a pigmented network, arborizing thin vessels, and hypopigmented unstructured areas on a pinkish background in the plaque area, while the nodular portion exhibited a dotted vascular pattern and white streaks.

Regarding our cases presented in this report, patient 1 exhibited the multicomponent pattern described by Bernard et al [3]. In this case, fine arborizing telangiectasias and linear vessels could be seen over structureless depigmented areas, which were surrounded by a pinkish background with unfocused vessels. Despite the fact that a pigmented network was reported in $87 \%$ of the lesions [3], in patient 1 there were no pigmented structures. Of interest, in patient 2 the only dermoscopic findings were a pigmented network with a pinkish background and no vessels could be observed. Although this case clinically had a morphea-like aspect, our dermoscopic features differed from those described by Costa et al [11] in their morphea-like variant in that we did not observe hypopigmented structures or vessels. Therefore, 
even in the same DSFP clinical variant, the dermoscopic features may vary.

In the attempt to summarize previously published data with the 2 new cases presented in this report (Table 2), we found that the 3 most common dermoscopic findings were the presence of vessels $(81 \%)$, followed by a pigmented network $(78 \%)$ and a pinkish background (66\%). Vascular structures included arborizing, mesh-like, linear-irregular, or polymorphic vessels. In relation to the pigment network observed, it corresponds to the increased pigment in the epidermal basal cells, rather than melanocytic proliferation. Therefore, it is an exception to the rule that the presence of a pigment network is a dermoscopic clue for the diagnosis of a melanocytic lesion [18].

Furthermore, 50\% displayed structureless hypo- or depigmented areas and $44 \%$ presented structureless light brown areas. Shiny white streaks, which were observed in more than half of the cases (53\%), may have been underestimated since this finding is only seen under polarized dermoscopy [20]. Unfortunately, not all previous studies described the dermoscopic acquisition method. This is an interesting issue to evaluate in future cases, since shiny white streaks have been related to malignant neoplasms, such as melanomas and basal cell carcinomas [20] and, therefore, could also be an indicator of DFSP.

Surgical excision remains the mainstay treatment for DFSP. Because of its infiltrating growth pattern, a wide excision with margins of at least $3 \mathrm{~cm}$ of clinically uninvolved skin is strongly advised for the treatment of DFSP [13,21,22]. Alternatively, Mohs micrographic surgery has become the treatment of choice for most skin cancers on the head and neck, as well as for recurrent or histologically aggressive lesions $[1,12,23]$. Adjuvant radiotherapy may be indicated when the resection margins are positive or when excision may result in significant aesthetic or functional damage [21]. Based on the knowledge about the signaling mechanisms of tumor proliferation, imatinib mesylate has shown significant therapeutic value in the treatment of DFSP [1,12]. Furthermore, predictors of poorer overall survival include older age at diagnosis, male sex, and large tumor size [15]. In addition, the pathological fibrosarcomatous variant is associated with an aggressive clinical course, with a higher rate of local recurrence and metastasis [24]. Since recurrences usually occur within 3 years of primary excision, patients should be followed up every 6 months during this period and annually thereafter [21].

\section{Conclusions}

This current report illustrates dermoscopic features of 2 cases of DFSP, as well as a review of previous reports. Our findings
Table 2. Most Common Dermoscopic Findings of DFSP and Their Frequency (Total of 32 Cases)

\begin{tabular}{|l|c|}
\multicolumn{1}{|c|}{ Dermoscopic Features $^{\mathrm{a}}$} & Frequency (n) $^{\text {Pigmented network }}$ \\
\hline Vessels & $78 \%(25)$ \\
\hline Structureless light brown areas & $81 \%(26)$ \\
\hline Shiny white streaks & $54 \%(14)$ \\
\hline Pink background & $63 \%(17)$ \\
\hline $\begin{array}{l}\text { Structureless hypo- or } \\
\text { depigmented areas }\end{array}$ & $56 \%(21)$ \\
\hline
\end{tabular}

a Bernard et al [3].

corroborate the earlier findings of most studies [3-,5,9-11]; however, atypical dermoscopic presentations have also been described [6-8]. Since this tumor may mimic benign lesions, dermoscopy is a valuable tool, indicating the need for a prompt biopsy and contributing to the correct diagnosis.

\section{References}

1. Li Y, Wang C, Xiang B, Shen S, Li L, Ji Y. Clinical features, pathological findings and treatment of recurrent dermatofibrosarcoma protuberans. J Cancer. 2017;8(7):1319-1323.

2. Thway K, Noujaim J, Jones RL, Fisher C. Dermatofibrosarcoma protuberans: pathology, genetics, and potential therapeutic strategies. Ann Diagn Pathol. 2016;25:64-71.

3. Bernard J, Poulalhon N, Argenziano G, et al. Dermoscopy of dermatofibrosarcoma protuberans: a study of 15 cases. $\mathrm{Br} J$ Dermatol. 2013;169(1):85-90.

4. Avilés-Izquierdo JA, Conde-Montero E, Barchino-Ortiz L, Lázaro-Ochaita P. Dermoscopic features of dermatofibrosarcoma protuberans. Australas J Dermatol. 2014;55(2):125-127.

5. Esdaile BA, Matin RN. Residents' corner August 2014. Clues in DeRmosCopy: dermoscopic features to aid earlier diagnosis? Eur J Dermatol. 2014;24(4):518-519.

6. Güngör S, Büyükbabani N, Büyük M, Tarıkçı N, Kocatürk E. Atrophic dermatofibrosarcoma protuberans: are there specific dermatoscopic features? J Dtsch Dermatol Ges. 2014;12(5):425-427.

7. Ehara Y, Yoshida Y, Shiomi T, Yamamoto O. Pigmented dermatofibrosarcoma protuberans and blue naevi with similar dermoscopy: a case report. Acta Derm Venereol. 2016;96(2):272-273.

8. Hartmann F, Haenssle HA, Seitz CS, Kretschmer L, Schön MP, Buhl T. Maculonodular lesion on the back of a 66-year-old man. Hautarzt. 2016;67(10):845-847.

9. Piccolo V, Russo T, Staibano S, et al. Dermoscopy of dermatofibrosarcoma protuberans on black skin. J Am Acad Dermatol. 2016;74(6):e119-e120.

10. Venturini M, Zanca A, Manganoni AM, Pavoni L, Gualdi G, Calzavara-Pinton P. In vivo characterization of recurrent dermatofibrosarcoma protuberans by dermoscopy and reflectance confocal microscopy. J Am Acad Dermatol. 2016;75(5):e185-e187.

11. Costa C, Cappello M, Argenziano G, Piccolo V, Scalvenzi M. Dermoscopy of uncommon variants of dermatofibrosarcoma protuberans. J Eur Acad Dermatol Venereol. 2017;31(8):e366e368. 
12. Acosta AE, Vélez CS. Dermatofibrosarcoma protuberans. Curr Treat Options Oncol. 2017;18(9):56.

13. Molina AS, Duprat Neto JP, Bertolli E, et al. Relapse in dermatofibrosarcoma protuberans: a histological and molecular analysis. J Surg Oncol. 2018;117(5):845-850.

14. Kreicher KL, Kurlander DE, Gittleman HR, Barnholtz-Sloan JS, Bordeaux JS. Incidence and survival of primary dermatofibrosarcoma protuberans in the United States. Dermatol Surg. 2016;42 Suppl 1:S24-S31.

15. Criscito MC, Martires KJ, Stein JA. Prognostic factors, treatment, and survival in dermatofibrosarcoma protuberans. JAMA Dermatol. 2016;152(12):1365-1371.

16. Al Barwani AS, Taif S, Al Mazrouai RA, Al Muzahmi KS, Alrawi A. Dermatofibrosarcoma protuberans: insights into a rare soft tissue tumor. J Clin Imaging Sci. 2016;6:16.

17. Lyu A, Wang Q. Dermatofibrosarcoma protuberans: a clinical analysis. Oncology Lett. 2018;16(2):1855-1862.

18. Soyer HP, Argenziano G, Chimenti S, Ruocco V. Dermoscopy of pigmented skin lesions. Eur J Dermatol. 2001;11(3):270-276.

19. Arevalo A, Altamura D, Avramidis M, Blum A, Menzies S. The significance of eccentric and central hyperpigmentation, multifo- cal hyper/hypopigmentation, and the multicomponent pattern in melanocytic lesions lacking specific dermoscopic features of melanoma. Arch Dermatol. 2008;144(11):1440-1444.

20. Shitara D, Ishioka P, Alonso-Pinedo Y, et al. Shiny white streaks: a sign of malignancy at dermoscopy of pigmented skin lesions. Acta Derm Venereol. 2014;94(2):132-137.

21. Saiag P, Grob JJ, Lebbe C, et al. Diagnosis and treatment of dermatofibrosarcoma protuberans: European consensus-based interdisciplinary guideline. Eur J Cancer. 2015;51(17):2604-2608.

22. Wollina Uwe, Langner D, Schönlebe J, França K, Lotti T, Tchernev G. Dermatofibrosarcoma protuberans: retrospective single center analysis over 16 years. J Med Sci. 2018;6(1):35-37.

23. Lowe GC, Onajin O, Baum CL, et al. A Comparison of Mohs micrographic surgery and wide local excision for treatment of dermatofibrosarcoma protuberans with long-term follow-up: the Mayo Clinic experience. Dermatol Surg. 2017;43(1):98-106.

24. Liang CA, Jambusaria-Pahlajani A, Karia PS, Elenitsas R, Zhang $\mathrm{PD}$, Schmults CD. A systematic review of outcome data for dermatofibrosarcoma protuberans with and without fibrosarcomatous change. J Am Acad Dermatol. 2014;71(4):781-786. 